because it is a well-known fact that inoculations made from the contents of the vesicles, etc., will cause the disease.

In the case of scarlet fever the lesion is undoubtedly in the throat, and the mucous surfaces leading therefrom. The discharges from these surfaces are highly infectious, as well as discharges from any sores on the face or other situations, which are evidently produced by direct infection from the nasal or aural passages.

I am not going to urge that there is no infection in the peeling cuticle, because it may receive infection indirectly in many ways, but in my opinion the desquamation which occurs, apart from the above discharges, and after the partaking of repeated disinfecting baths, plays little or no part in spreading the infection of this disease.

It would appear that scarlet fever is a disease of the working rather than the middle and upper classes. I have got out some particulars in this respect for Halifax for five years, Igor to I905, and the following table, No. 9, shows the results :-

Attack rate per $\mathrm{I}$, ooo of persons living in different sized houses. Average of five years, I. gor -5 :-

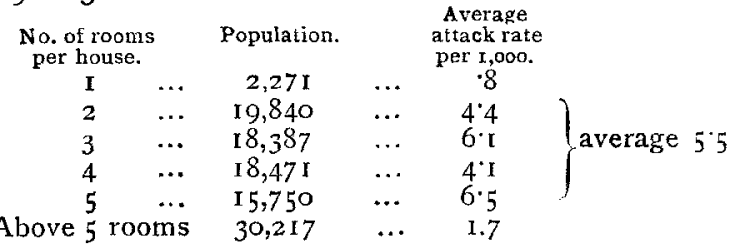

It will be observed that the attack rate is very low among persons living in single-roomed houses, from the obvious reason that very few susceptible persons live in that class of house ; but taking houses with from two to five rooms the average attack rate was $5^{\circ} 5$ per $I, 000$, against only $I^{\prime} 7$ per 1,000 in houses with more than five rooms.

I fear that I have already detained you too long upon this subject, and will therefore bring my remarks to an end.

In conclusion, it appears to me that we have yet a great deal to learn regarding this disease, the elucidation of which would involve a prolonged and careful study, and an intricate and elaborate bacteriological investigation. The inquiry would, in my opinion, have to be conducted not only during epidemic years, but also during non-epidemic periods; and the time required to carry out the necessary investigations would be too great and too prolonged to make it possible of achievement by a medical officer of health in active work.
THE SYSTEMATIC EXAMINATION OF THE EYES OF SCHOOL CHILDREN.* By N. BISHOP HARMAN, M.A., M.B. Cantab, F.R.C.S, Eng,

Lecturer in Ophthalmology to the West London PostGraduate College; Assistant Ophthalmic Surgeon to the West London Hospital ; Ophthalmic Surgeon to the Belgrave Hospital for Children; Oculist to the London County Council Blind Schools.

TO tell you all that the title of my lecture implies in the brief space of one hour would, I think, pass the wit of man. What I propose to do is to take you in imagination into any large school, say in London, and there show you from the results of my experience what eye conditions you are most likely to find amongst the children, and how you can best set about finding out these conditions.

In entering any school the first person whose acquaintance the school doctor should make is the head-master. The friendly help of the chief of the school is of the greatest advantage to the doctor, for a good master can give information as to the character and capabilities of a child which is scarcely second, and is often superior, to that which can be obtained from the parent, and from this source the doctor can gain information that would be lost to his unaided effort. In the early days of school inspection $I$ can remember that the doctor's reception was not always friendly; his visit was conceived to be an additional worrying inspection forced on an already over-inspected school staff; but by this time the teachers have learned to regard the doctor as a friendly helper, and they welcome and invite his assistance, even if he occasionally has to criticise.

The next procedure which I have found of profit is to make a tour of the school when all the children are in their places and to take note of the conditions of the class-rooms as regards their lighting, natural and artificial, and their ventilation; the arrangement of desks and the attitude of the children; the state and position of blackboards, diagrams, and maps; the quality of the print in the books in use; and the style of writing, drawing, and needlework taught, and the attitude of the children during these exercises. All these things come within the sphere of influence of the doctor who is investigating eyes.

\footnotetext{
*A lecture delivered at the Rooms of the Society of Medical Officers of Health, J anuary izth, Igog.

Note.-The lecture was illustrated by many diagrams and figure which cannot be reproduced. They will be found, together with much other work on school conditions, in "The Conjunction in Health and Disease," by N. Bishop Harman. Balliere, Tyndall \& Co., London.
} 
As each class-room is visited; so a preliminary examination of the children is made; the doctor passes through the passages between the desks and each child is required to look straight into his eyes, and at any indication of a departure from the normal the child is marked for further examination.

Besides the class-rooms, the eye doctor should examine the lavatories of the school, particularly the wash-places, and note the condition and number of the towels, and ascertain the frequency with which they are renewed. The reasons for these points will appear later.

Classification of Eye Conditions.

Taking the eye conditions as a whole, we shall find that they are divisible into two great groups: (I) External diseases; (2) Functional disorders. Each of these groups has points of importance, and although for the sake of convenience we separate them, it must be always kept in mind that the association of external disease and functional disorder is most intimate, and often casual, in relation.

\section{I.-External Diseases.}

Recently I examined a number of English children in the schools of the Hackney district of London. The region includes all varieties of social conditions: good residential quarters at Clapton, artisan dwellings at Homerton, and crowded slum regions at Bethnal Green. The district is representative of a great city, and the conditions found in these schools are a fair average of what you may expect to find.

The examination was made during the months of July and November of one year, and covered thirty schools, with 2 I, 893 children of ages from seven to thirteen years. Subsequent to the examination the schools were classified according as I judged the situation of the region, the housing, and the condition of the children favourable to their well-being. Thirteen schools were found in which the children reached a fair average of cleanliness, three schools were above the average, and fourteen below. The following results appeared :-

\begin{tabular}{|c|c|c|c|}
\hline CLEANLINESS. & & CASTES OF DISEASE. & PERCENTAGE. \\
\hline $\begin{array}{l}\text { Above average } \\
\text { Average ... } \\
\text { Below average }\end{array}$ & $\begin{array}{l}\cdots \\
\cdots \\
\cdots\end{array}$ & $\begin{array}{r}19 \text { in } 2,174 \\
134 \text { in } 9,463 \\
197 \text { in } 10,256\end{array}$ & $\begin{array}{l}0.873 \\
1.416 \\
I .92\end{array}$ \\
\hline Totals .... & ... & 351 in 21,893 & $1 \cdot 608$ \\
\hline
\end{tabular}

We may say that roughly 2 per cent. of elementary school children are subject to some condition of eye disease due to a present inflammation, or to one that is past, and that we shall find twice as much eye disease amongst the poorly-kept section of the population as amongst the well-kept.

The diseases found were as follows :-

Per cent.

$\begin{array}{llllll}\text { Conjunctivitis } & \ldots & \ldots & \ldots & \ldots & \text { IO }_{5}\end{array}$

$\begin{array}{llllll}\text { Blepharitis } & \ldots & \ldots & \ldots & \ldots & 74^{\circ} \mathrm{O}\end{array}$

$\begin{array}{llllll}\text { Phlyctenulae } & \ldots & \ldots & \ldots & \ldots & I_{5} 5^{\circ} \mathrm{O}\end{array}$

Effects of ophthalmia neonatorum $\quad \ldots \quad$ I'5

$\begin{array}{lllll}\text { Interstitial keratitis } & \ldots & \ldots & \ldots & \mathrm{r} \cdot 5\end{array}$

Congenital anomalies $\ldots \quad \ldots \quad \ldots \quad \ldots \quad 4^{\circ} \circ$

$\begin{array}{llllll}\text { Eyes lost } \ldots & \ldots & \ldots & \ldots & \ldots & 2 \cdot 0\end{array}$

BLEPHARITIS.

Sore eyelids account for the bulk of the cases. This is an inflammation of the margins of the lids, and involves the lashes, their glands, the Meibomian glands, and the strips of skin and conjunctiva bordering the lid margins.

Investigating the cases of blepharitis at the Belgrave Hospital for Children for ten years, I found that the maximum incidence occurred at the age of four years; from then until the end of the school years there was a steady flow of cases, with a second but lesser peak of frequency at the age of seven years.

Blepharitis is essentially a dirt disease. It is true there are contributory causes other than dirt, but the disease scarcely occurs in clean children, even when these contributory causes are of equal weight. At the age of four the infants in the Belgrave region entered the infant departments, dirt and microbes had a cumulative effect. Again, at Moorfields Hospital, I found the dirtier alien patients suffered out of all proportion to their numerical standing.

Contributory causes to the production of the disease are the exanthemata and eye strain. Measles is a particularly frequent starting-point for sore eyelids : the conjunctivitis set up by the fever leaves the lid margins sodden, and a ready culture medium for organisms of which there are always more than enough about the lashes of the lids. The frequency of the fever amongst infants may account in part for the maximum incidence at the age of four years.

Next to this, eye strain may be held a contributory cause, and if it be less the cause of the onset of acute cases, it is certainly one of the most frequent in chronic cases. If the doctor will examine a class, say of girls before and after an afternoon's needlework, he will find a striking difference in the appearance of the eyelids of a number of the girls. At the 
close of the lesson he will find that an appreciable number have reddened margins to their lids, and he will notice that the rate of blinking is distinctly increased. We are familiar with the never-ending work of our hearts, and the automatic nerve centres, but we scarcely give a thought to the almost equally arduous labour of the eyelids. In the space of a single working day their margins will come in contact several thousand times, more than enough to produce rawness of them were they not specially protected. Their protection lies in the layer of protecting grease poured on to the lid margins from the Meibomian glands. When the blinking is excessive, as it is when the vision is defective or when the work forced upon the eye is too severe, the protection of the grease is insufficient, the margins become sore; and they are further infected by the manner in which the child rubs its tired eyes. When the lids are inflamed the good quality of the Meibomian grease is lost, and is no longer protective; so a vicious circle of circumstances is started, and blepharitis is in a fair way to become chronic. Only scrupulous cleanliness of person can prevent these effects if the causes be left untended, and the longer they last the more difficult is the cure.

The treatment is simple enough: Clean the lids, rub them vigorously and frequently with a warm alkaline lotion (Sod. bicarb. 5 per cent.) until the margins are completely free from crusts, then rub in a mild mercurial ointment, such as the yellow oxide, of I per cent. As soon as possible correct any error of refraction with appropriate glasses.

Phlyctenular Conjunctivitis.

This came next in order of frequency as the cause of eye trouble with I 5 per cent. It must be remembered how serious is this inflammation in its lasting effects on the transparency of the cornea, and consequently on visual acuity. It is a disease of the poor and of young children. Cases are very rare in the first year of life; the maximum incidence falls from four to six years, when the milk teeth are decaying. The children very commonly suffer from chronic coryza, sores about the nostrils and lips, eczema of the face, or perhaps discharging ears. They are invariably ill-fed. At Moorfields Hospital I found the disease much less frequent amongst Jewish children than native. Jews feed their children well, and use much oil in cooking; we may note here that cod-liver oil is the best cure for the disease. I cannot enter at length into the cause of the disease, but I may say that I have put forward evidence to show that it is not the effect of a specific microbe, but that it is an efflorescence or herpetiform eruption on the terminals of the first and second division of the fifth cranial nerve caused by peripheral irritation of collateral branches of the second division of that nerve, by septic teeth, and nasal and labial sores, in ill-nourished children. The disease accounts for more cases of damaged eyes, and therefore of diminished vision, than any other ; and no better argument for instruction in oral cleanliness and dental clinics can be made than the citation of this disease.

Acute Conjunctivitis.

This accounted for ro per cent. of the cases. Most of these appeared to be due to infection with the Koch-Weeks bacillus. Every school doctor should be thoroughly familiar with the characters of this organism, of the MoraxAxenfeld bacillus, and of the Xerosis bacillus. The first two are conjunctival organisms almost exclusively, and consequently the man who has taken out a course of bacteriology may be quite unacquainted with them, the more especially as they do not readily take on the saprophytic life of the laboratory, and cannot be "kept in stock." The organisms are quite characteristic, and are easy of distinction in film preparations, and I would refer you to the descriptions and pictures of them to be found in the book referred to in the footnote on the first page of this paper.

Trachoma, that scourge of Eastern countries, was extremely rare; only two certain cases and one doubtful case was found, or less than I per cent. of the diseased conditions. I would warn you of mistaking simple follicular hypertrophy, which is very common amongst school children, especially girls, for trachoma. Follicular hypertrophy is quite innocuous, but it is a useful indication of a general poverty of health. The follicles are mainly situated in the conjunctiva of the lower lid, and they do not cause any symptoms. Trachoma is essentially a chronic overgrowth of the stroma of the conjunctiva, and is manifested in a roughening of the usually smooth and shining conjunctiva of the upper lid; and it is associated with a series of distinct and distressing symptoms both subjective and objective. Do not miss everting and examining the upper conjunctiva of any child whose lids look heavy and drooping, for this sign forms the earliest and most distinct of the objective symptoms. 
Discharging Eyes.-Children with discharging eyes should be excluded from school; notice of the exclusion being given to the proper authority so that treatment may be effected. This is particularly important when epidemics occur.

There is no more fruitful mode of spreading eye infections than a promiscuous use of towels. In residential schools each child should have exclusive use of his own towel. The towel and other toilet appliances, as facerubber or sponge and tooth brush, should be hung on a hook and marked with the number of the child. In schools where these regulations are enforced, "school ophthalmia" is almost a thing of the past. In day schools the matter is more difficult: it is not possible to supply towels for each scholar. We best get over the difficulty by a rule allowing the use of wash-basin and towels for hands only, and prohibiting the washing of faces at school. Dirty children should be sent home to wash. Should an outbreak of conjunctivitis appear in a day school, the towels should be withdrawn from use. In an epidemic of mucopurulent catarrh due to the Koch-Weeks bacillus, which I investigated, the spread of the epidemic was checked when the issue of towels was stopped.

DirT ANd Disease.

Enough has been said to show how close and constant is the association of these two conditions. The incidence of disease, the occurrence of bacteria on the conjunctiva and on the lid margins, and the frequency of follicular hypertrophy, all show that germs, disease, or at least unhealthy conditions, are caused and increased by dirt. The school doctor will therefore inevitably find himself as much the opponent of dirt as of disease, and in effect become a teacher of the essentials of hygiene-cleanliness.

\section{II.-Functional Disorders.}

Disorders of vision are by far the most frequent source of trouble amongst school children. Of 30,000 London school children 26 per cent. were found to have defective visual acuity (Dr. Kerr's return, I903); over one in every four children could not pass the test of reading $6 / 9$ on Snellen's card. The conditions were worse in the poorer, overcrowded districts, for there 30 per cent. failed, and amongst the alien Jews of the East End 36 per cent. were defective. Again, girls have rather worse vision on the average than boys. In one goodclass school I found I 8 per cent. deficiency in boys and 26 per cent. in girls.
The effect of social environment and of sex on vision, or, it may be said, on the growth of the eye, is striking. To some extent this may be due to the early age at which poor children and girls are set to work at home, before and after school hours.

Wits and Sight.-In practical work it has been found again and again that "dull and stupid" children were so because they could not see; so, if only a half or a third of these school children who fail to pass the vision test have serious defect of vision, there is enough to account for much failure in school teaching.

Testing the Vision.*-It is sufficient in schools to take the vision of both eyes together, unless some exceptional condition should indicate the contrary. Snellen's test card should be exposed in good daylight, but not in direct sunlight. The letters should be pointed to by a teacher to help the attention of the child. If the light be poor, shorten the distance to such a length as will allow the doctor to read the 6/6 line with his accustomed ease. (It is to be supposed the doctor has good vision, or proper glasses !)

Children over ten years of age who cannot read $6 / 9$, and those from seven to ten years who cannot read $6 / 12$, should have their eyes examined.

If the vision of infants under seven years of age is to be tested, the $\mathbf{E}$ test card should be used. This is made up of different sized E block letters in various positions. The child is given a card with an $\mathbf{E}$ thereon, and is shown how to hold it in the position of the letter pointed to. A judicious bribe of "sweets" adds to the child's interest, and better results are obtained. As a rule it is sufficient in infants' classes to select for examination those children who squint or have sore eyes, and any whom the teachers suspect of seeing with difficulty.

The inability of young children to see $6 / 6$ does not necessarily imply poor vision, for there is in all these tests a mental factor: the greater the familiarity with the letters the more easily are they recognised.

\section{ERRORS OF REFRACTION.}

For the most part the errors amongst the younger children are those of hypermetropia with or without astigmatism. Amongst older children myopia increases, especially amongst those who are astigmatic. The condition of

* In London this is done in the first instance by the teachers, who enter the results in a special column of the attendance register. This is a great saving to the doctor. 
the eye can only be determined by retinoscopy after the use of a proper cycloplegic. I would warn you against putting any serious value upon the results of retinoscopy in children when no efficient cycloplegim is used. By careful comparative tests in a large number of cases, I have found that in just those cases where one desires a useful indication of the cause of the defect in vision one is led astray; for the mere flashing of a light into the eyes of a young child tends to set up a spasm of the ciliary muscle, and so a child may appear to be myopic when it is not. Atropine or hymatropine must be used. The former is best for home use as an ointment of I per cent. thrice daily for a week. But when the surgeon desires quickly to paralyse the ciliary muscle, the use of the pure alkaloids of homatropine and cocaine ( 2 per cent. of each dissolved in castor oil by gentle heat) gives excellent results. A drop should be placed in each lower farnix, and the eyes lightly bandaged; after half-an-hour the dose should be repeated, and with the completion of the full hour the eyes will be ready. The effect of the drug will pass off in twenty-four hours, but can be neutralised in thirty minutes by the installation of Eserine I per cent. in castor oil.

School Conditions Affecting Eyes.

Vision depends upon the detection of contrasts, good light is necessary to show up the contrasts. An adult can read letters in a poor light because he is familiar with the letters; a child has not this familiarity, so needs an ample light.

Windows should be large, filling nearly the whole of the children's left-hand wall of the room, right up to the ceiling; then the desks will be well lighted, and the shadow of the writing hand will not obscure the writing. Artificial illuminations at each desk should not be less than ten metre candles, i.e., the light given by ten standard candles at the distance of one metre. It should be steady, and white in colour.

Desks.-The seat should be of such a height that the child's thighs rest upon it while the feet are on the ground; the desk height should be such that when the child sits erect the flexure of the elbow is just on a level with the edge. The desk should slope $\mathrm{I}_{5}$ deg. down towards the child, whilst for reading the book should be tilted on a frame to $50 \mathrm{deg}$.

Print.-The greatest contrast is obtained by leaded lines, i.e., well-spaced lines, of not more than four inches in length, and with heavy black type of the "Clarendon" style.

\section{This is Double Pica.}

\section{This is Pica, the smallest School Type.}

In the infants' classes under five years the smallest type should be double pica, and in the upper standards the type should not be smaller than pica. The paper should be hard pressed, but not highly glazed. No print should be held nearer to the eyes than twelve inches.

Writing.-Upright writing with a broad pen is the best from all points of view. Slates should be abolished: the contrast of writing and background is poor; and they are dirtychildren will spit on them.

Blackboards.-The surface should be of a good dull black, and frequently renewed to prevent "shine." The board should be placed on the children's right-hand side. The letters marked on the board should not be smaller than two inches square (Snellen's D. 36).

Needlework should be excluded from infant classes; wool-work should be taught instead. In the first lessons on needlework, white calico and black sewing cotton with large needles should be used. Fine needlework should not be permitted in school. Should any girl excel in fine needlework she should be medically examined lest she be myopic!

Myopes.-Fine art; particularly needlework, should be prohibited in any degree of myopia exceeding I dioptre. Knitting should replace sewing, and the child should learn to do it blindfold. Myopes of $6 \mathrm{D}$. and more should in most cases be provided for in special schools.

Spasm and Failure of Accommodation.-In over-worked and under-fed children there may be complaint that "the print suddenly goes misty" - there is failure of accommodation. In some cases they also temporarily fail to see in the distance-there is spasm of accommodation. Careless examination of these latter cases may mistake them for myopes, and great harm may result from the erroneous prescription of minus lenses.

Squint.-The urgency of proper treatment of these cases cannot be too much insisted upon. Children do not "grow out of squint," but the squinting eye will surely go blind. 